International Journal of Computer Science \& Information Technology (IJCSIT) Vol 3, No 4, August 2011

\title{
CULTURAL INFLUENCES ON EXPLICIT AND IMPLICIT KNOWLEDGE SHARING BEHAVIOUR IN VIRTUAL TEAMS
}

\author{
Xi Zhang ${ }^{1,2}$ \\ ${ }^{1}$ Institute of Policy and Management, Chinese Academy of Sciences, P. R. China \\ ${ }^{2}$ Center for Innovation and Development, Chinese Academy of Sciences, P. R. China \\ xizhang@casipm.ac.cn
}

\begin{abstract}
Knowledge can be classified as explicit and implicit knowledge. This study investigates how national or regional culture influences on the explicit and implicit knowledge sharing behaviour in the context of multi-national virtual teams. The findings suggest national culture significantly influence on participants' attitude to sharing knowledge in IT-enabled virtual teams. This study provides some suggestions for the virtual team management.
\end{abstract}

\section{KEYWORDS}

National Culture, Hofstede's Dimensions, Virtual Teams, Knowledge Contribution.

\section{INTRODUCTION}

As organizations are geographically dispersed nowadays, numerous organizations have changed to virtual organizations, or virtual teams which adopted information systems, especially knowledge management systems (KMS) for supporting knowledge sharing (KS) behavior among their members [1,2]. In virtual teams, knowledge sharing can be defined as individual sharing work relevant experiences and information with other team members $[1,2]$. KMS provides an IT-based platform where employees from different places can communicate and share knowledge. However, contrary to earlier expectations, these firms face the serious problem of "KS dilemma" where people are unwilling to share knowledge through these ITbased KMS [3, 4]. Several studies have found that the availability of ICT is no guarantee of KS [5-7]. Some studies have found the "KS dilemma" to be even more salient in an IT-based environment [3].

A considerable body of KS literature has adopted the framework of social exchange theory (SET) to understand people's unwillingness to share knowledge in KMS. According to SET, one of most critical reasons for unwillingness to share knowledge is that people are not well motivated to share their knowledge [8]. Based on SET, people can be motivated by motivators such as extrinsic and intrinsic factors [9, 10]. Since individuals' motivations can be influenced by organizational interventions, some supporting strategies are considered to increase employees' KS behavior [9]. However, a review of the KS literature shows these supporting strategies affect employee KS behaviors, especially in the IT-based environment, at dissimilar 
International Journal of Computer Science \& Information Technology (IJCSIT) Vol 3, No 4, August 2011

levels $[11,12]$. These supporting strategies are also interacted with individual differences, and members' cultural context [13].

In the IT-based virtual teams, rare empirical studies have examined the effect national cultural influences and individual differences jointly on KS behaviour. It is still to be investigated whether some motivations are effective or not in encouraging KS behavior in the IT-based environment with different individuals under different cultures. This study tries to fill this gap that we investigate the extrinsic and intrinsic motivations on knowledge sharing in the multinational virtual teams with three different cultures (mainland China, Hong Kong, and Netherlands) and two different individual orientations.

This study contributes to knowledge sharing literature in two ways. First, we examine the relationship between motivations and knowledge sharing behavior in the context of virtual teams under different cultures (i.e., Hofstede's four culture dimensions). Research has suggested that members' national cultural orientation is the important antecedent of knowledge sharing behavior [7, 9]. Second, our findings may have some implications for the management of virtual team leaders when they make decision on selecting member and applying incentive mechanism on KM.

\section{LITERATURE REVIEW}

\subsection{Cultural Influences on Knowledge Sharing}

Although some KM technologies have been applied to support KS in KMS, they may not ensure the KS will occur $[14,15]$. There has been increasing interest in the impact of national culture in information systems and knowledge sharing research. Traditionally, most of these studies rely on some or all of Hofstede's dimensions [16-18]. However, researchers have begun to criticize this approach and have adopted new perspectives on national culture. For example, Myers \& Tan suggest that the fundamental assumption upon which Hofstede's work is based is questionable and propose a more dynamic view of culture. Walsham also argues that structurational analysis provides a deeper examination of cross-cultural working than is found in the Hofstede-type studies. Weisinger \& Trauth present how the theoretical approach of situating culture contributes to a better understanding of contextualism in the cross-cultural IT environment. These new perspectives are worthwhile and fruitful. However, it does not mean that Hofstede framework is no longer useful. Srite \& Karahanna provide an excellent example. Based on Hofstede's four dimensions, they explore the role of espoused national cultural values in technology acceptance. The four dimensions are:

- Power distance: Power distance is defined as "the extent to which the less powerful members of institutions and organizations within a country expect and accept that power is distributed unequally"[19].

- Uncertainty Avoidance: Hofstede [19] defines uncertainty avoidance as "the extent to which the members of a culture feel threatened by uncertain or unknown situations." Disregard of accuracy has been described as one of the characteristics of Chinese people in terms of feeling less need to be exact and precise [20].

- Individualism: Collectivism pertains to societies in which people from birth onwards are integrated into strong, cohesive in-groups, which throughout people's lifetime continue to protect them in exchange for unquestioning loyalty [19]. 
International Journal of Computer Science \& Information Technology (IJCSIT) Vol 3, No 4, August 2011

- Concern for Face: Saving face is a by product of collectivism that, in China, is very deeprooted and influential.

\subsection{Individual Influences on Knowledge Sharing}

The relationship between perception of motivations and social behavior depends on individuals' exchange orientation [13]. Eisenberger et al. [21] were the first to explore individual exchange orientation, called "individual exchange ideology" (ID). ID is the preexisting belief related to personal value [22]. According to the definition of Eisenberger et al. [21], ID is concerned with the extent to which individuals believe that their own reciprocating behavior (e.g. KS) should be a function of the benefits they receive from the social exchange.

Eisenberger et al. [21] claim that high ID employees will be more likely to positively respond to extrinsic benefits relative to low ID employees. They argue that extrinsic benefits signify effort-benefit expectancy for high ID employees. To exchange more anticipated extrinsic benefit, high ID employees will be motivated to input more work efforts. The KS literature denotes that KS involves a set of costs (e.g., work effort and loss of power), thus organizations should provide various extrinsic benefits to motivate employees' KS [9]. According to the logic of Eisenberger et al.'s [21] argument, for employees with ID, higher reward connotes higher net benefits of KS and will lead to higher levels of KS behavior. For employees with low ID, higher reward will not increase their KS, as their KS behavior is due to their morals and sense of obligation rather than to extrinsic benefits. Based on the above argument, the positive effect of reward on KS behavior is expected to be stronger for people with high ID than for people with low ID.

However, there has been mixed evidence about moderating effects of ID in social interactions. Especially in the more recent literature, research has reported extremely ambiguous results on the interaction effect between ID and benefits (e.g., rewards). Some recent studies have even found negative moderating effects of ID. Redman et al. [23] suggest that the moderating effects of ID may be contingent on the different contexts. In other words, organizational contextual factors may act as a third-level moderator to regulate the interactive effect of reward and ID on employees' reactions.

SET does not consider the influence of organizational and national contexts [13]. Especially in IT-based environments (e.g., KMS), knowledge contributors' efforts in sharing knowledge may not be easily identified. In this study's background, the influence of environmental factors on the interactions of ID and rewards are more significant. This requires adopting the national cultural perspective to understand the explicit and implicit knowledge sharing in the virtual teams.

\subsection{Explicit and Implicit Knowledge Sharing}

Knowledge can be defined as different types. Nonaka et al. [24] classify knowledge into explicit knowledge and tacit knowledge. Explicit knowledge is codified knowledge that has been articulated in symbolic form; tacit knowledge includes two elements: 1) the cognitive element referring to mental models (e.g., beliefs and viewpoints), and 2) the technical element referring to skills that can be applied in a special context (e.g., know-how). According to Alavi et al. [1], IT is more useful for supporting explicit KM rather than for tacit KM. Some researchers also identify a third dimension of knowledge, namely, implicit knowledge [25]. Nichols [25] purports that knowledge which has not been articulated should be classified as 
International Journal of Computer Science \& Information Technology (IJCSIT) Vol 3, No 4, August 2011

implicit knowledge and tacit knowledge. Implicit knowledge can be articulated, while tacit knowledge cannot be articulated. The research context in this study is IT-supported virtual teams to support knowledge sharing where knowledge is classified into two dimensions: implicit and explicit knowledge. Thus, in this study, explicit knowledge is knowledge that exists in symbolic or written form (e.g., proposals, reports, or knowledge obtained from other publications) [26]. Implicit knowledge is knowledge that can be expressed in verbal, symbolic or written form but is not yet expressed (e.g., know-how, know-where, know-whom, expertise) [26].

\subsection{Intrinsic and Extrinsic Motivations of Knowledge Sharing}

Numerous studies have investigated KS from the cost-benefit perspective [9, 10]. According to social exchange theory (SET), Kankanhalli et al. [9] have identified the social and individual cost and benefit factors in knowledge contribution in the electronic knowledge repository. Based on Kankanhalli et al.'s [9] framework, the benefits include extrinsic benefits (i.e., organizational reward, image and reciprocity), and intrinsic benefits (i.e., knowledge selfefficacy, enjoyment in helping others).

The benefits of KS in KMS refer to the resources which contributors can receive in KS [9, 10]. Extrinsic benefits are resources which contributors gain from other people [13]. Furthermore, extrinsic benefits can be divided into social benefits and economic benefits. The extrinsic benefits in sharing knowledge are summarized in the following:

- Organizational rewards: Organizational rewards include monetary incentives such as bonuses, and non-monetary awards such as job security [14]. One fundamental motivation of sharing knowledge is getting rewards for contributions [9, 27-29].

- Reciprocity: Reciprocity is a social benefit of individuals engaged in social exchange [30]. Reciprocity of KS refers to the belief that current knowledge contribution will lead to future help from others [10].

- Reputation: Contributors may receive intra-organizational respect for KS behavior [9].

Intrinsic benefits of KS in KMS are satisfaction or pleasure which contributors gain themselves $[9,13]$. Different from extrinsic benefits, intrinsic benefits strongly relate to the work itself [31]. There are several types of intrinsic benefits in sharing knowledge:

- Knowledge self-efficacy: In KS, self-efficacy refers to the confidence in their ability to provide valuable knowledge to organizations [32]. When people share knowledge, they will be intrinsically motivated with enhanced confidence [9, 32].

- Enjoyment in helping others: People are intrinsically motivated to share knowledge for the enjoyment they receive from helping others [10].

\section{HYPOTHESES DEVELOPMENT}

\subsection{National Culture Influence}

In this study, we still adopt Hofstede's framework to explore national culture impact. This framework is particularly advantageous for national culture research in software because it was 
International Journal of Computer Science \& Information Technology (IJCSIT) Vol 3, No 4, August 2011

based on a single, consistent control group: employees in dozens of international offices of IBM [33]. We examine four dimensions (power distance, uncertainty avoidance, masculinity and individualism) considered especially relevant to our research question and their impact on benefit-knowledge sharing relationship.

In this study, we will investigate three different regions, i.e., Beijing, Hong Kong, and Netherlands. The comparisons of cultural dimensions are illustrated in Table 1.

Table 1. Cultural Dimensions Comparison

\begin{tabular}{|l|l|l|l|l|}
\hline & Power Distance & Individualism & Masculinity & Uncertainty \\
\hline Beijing & 80 & 10 & 50 & 38 \\
\hline HongKong & 63 & 20 & 52 & 29 \\
\hline Netherland & 33 & 80 & 10 & 50 \\
\hline
\end{tabular}

Of note is Hong Kong's relatively low Uncertainty Avoidance (UAI) Dimension ranking of only 29, compared to an average of 63 for the Far East Asian countries. This very low level of Uncertainty Avoidance is the fourth lowest in the World, with only Denmark (23), Jamaica (13), and Singapore (8) having lower scores for this Dimension. The low Uncertainty Avoidance ranking indicates a society that not only tolerates uncertainty and a freedom of opinions, but uses this strength, in the case of Hong Kong, to be a place where many varying cultures and ideas can come together. Geert Hofstede describes people in a culture with the low UAI Dimension as, ". . . more phlegmatic and contemplative ..". In this cultural environment, participants will be positively drived from extrinsic motivations, without fairing uncertain situation if they share knowledge. The above argument is captured by the following hypothesis:

Hypothesis 1: Compared with Beijing and Netherland Participants, participants from Hong Kong will be more responsive to the extrinsic rewards with explicit and implicit knowledge sharing in the virtual teams.

Of note is Mainland China's significantly higher Power Distance ranking of 80 compared to the other Far East Asian countries' average of 60, and the world average of 55. This is indicative of a high level of inequality of power and wealth within the society. In the culture with high power distance, participants will response more positively to the intrinsic rewards (e.g., enjoyment in helping), as they will perceive helping others will improve the relationship between them. The above argument is captured by the following hypothesis:

Hypothesis 2: Compared with Hong Kong and Netherland Participants, participants from Beijing will be more responsive to the intrinsic rewards with explicit and implicit knowledge sharing in the virtual teams.

Netherland's highest Hofstede Dimension is Individuality (IDV) at 80, which ties with Canada as the fourth highest worldwide IDV ranking, behind the United States (91), Australia (90), and the United Kingdom (89). The high Individualism (IDV) ranking for the Netherlands is indicative of a society with more individualistic attitudes and relatively loose bonds with others. The populace is more self-reliant and looks out for themselves and their close family members. 
International Journal of Computer Science \& Information Technology (IJCSIT) Vol 3, No 4, August 2011

In this cultural environment, participants may follow their heart, without care about economic and social motivations. That is,

Hypothesis 3: Compared with Beijing and Hong Kong Participants, participants from Netherlands will not significantly response to the extrinsic and intrinsic motivations with explicit and implicit knowledge sharing in the virtual teams.

\subsection{Individual Influence}

Under the context of IT-based knowledge sharing (KS), when social loafing is high, individuals' sharing work is not identified. Employees will deem economic strategies (e.g., organizational reward) as a collective incentive rather than a reward to their knowledge contribution. High ID Individuals have a social loafing tendency to maximize their own net benefits, and respond to organizational reward with little knowledge contribution behavior. Low ID employees, for whom moral reasons or obligation induces their KS, organizational reward will not affect their KS. Therefore, it is expected that the interactive effect of ID and reward is negative. The above argument is captured by the following hypothesis:

Hypothesis 4: In the short-term virtual teams, interactions of economic strategies and individual exchange orientation are negatively related to explicit and implicit knowledge sharing behavior.

For the indirect effect of social loafing on the relationship between social factors and KS behavior, the short-term nature of virtual organization should be considered in calculating the social benefits of KS. In KS, the social benefits include not only the benefits of the current exchanges, but also some additional benefits of future exchange [34]. "Rational" individuals will compare the net benefits of all future exchanges with the cost. They may be willing to accept a less-than-hoped-for outcome in the short term because they believe that they will receive their desired outcomes over time. Thus, an individual with strong ID may show more work effort than the organization expected. In this condition, ID may have a positive influence on the relationship between social benefits and KS. However, in short-term teams, members will focus on the benefits from current exchange [35-37].

Most of virtual teams are short-term projects and have high social loafing environments. Although members may perceive high levels of short-term reciprocity in the project, "rational" individuals may also be concerned with the lack of future and additional benefits from longerterm exchanges. Based on the cost-benefit calculation, they will show a lower level of KS than the response to the benefits. In contrast, individuals with weak ID have general expectations on the benefits. They will share knowledge as a normal benefit of reciprocity. Thus, in this circumstance, it is expected that the interactive effect of individual orientation and social strategies are negative. The above argument is captured by the following hypotheses:

Hypothesis 5: In the short-term virtual team, interactions of social reciprocity benefits and individual exchange orientation are negatively related to explicit and implicit knowledge sharing behavior.

Hypothesis 6: In the short-term virtual team, interactions of social reputaion benefits and individual exchange orientation are negatively related to explicit and implicit knowledge sharing behavior. 
International Journal of Computer Science \& Information Technology (IJCSIT) Vol 3, No 4, August 2011

Different from extrinsic benefits, intrinsic benefits strongly relate to the work itself [31]. Thus, the relationship between intrinsic motivations and knowledge sharing behaviour may not be moderated by individual exchange orientation. The above argument is captured by the following hypotheses:

Hypothesis 7: In the short-term virtual team, interactions of self-efficacy and individual exchange orientation are not significantly related to explicit and implicit knowledge sharing behaviour.

Hypothesis 8: In the short-term virtual team, interactions of enjoyment in helping others and individual exchange orientation are not significantly related to explicit and implicit knowledge sharing behaviour.

\section{RESEARCH MODEL}

The research model is illustrated in Figure 1.

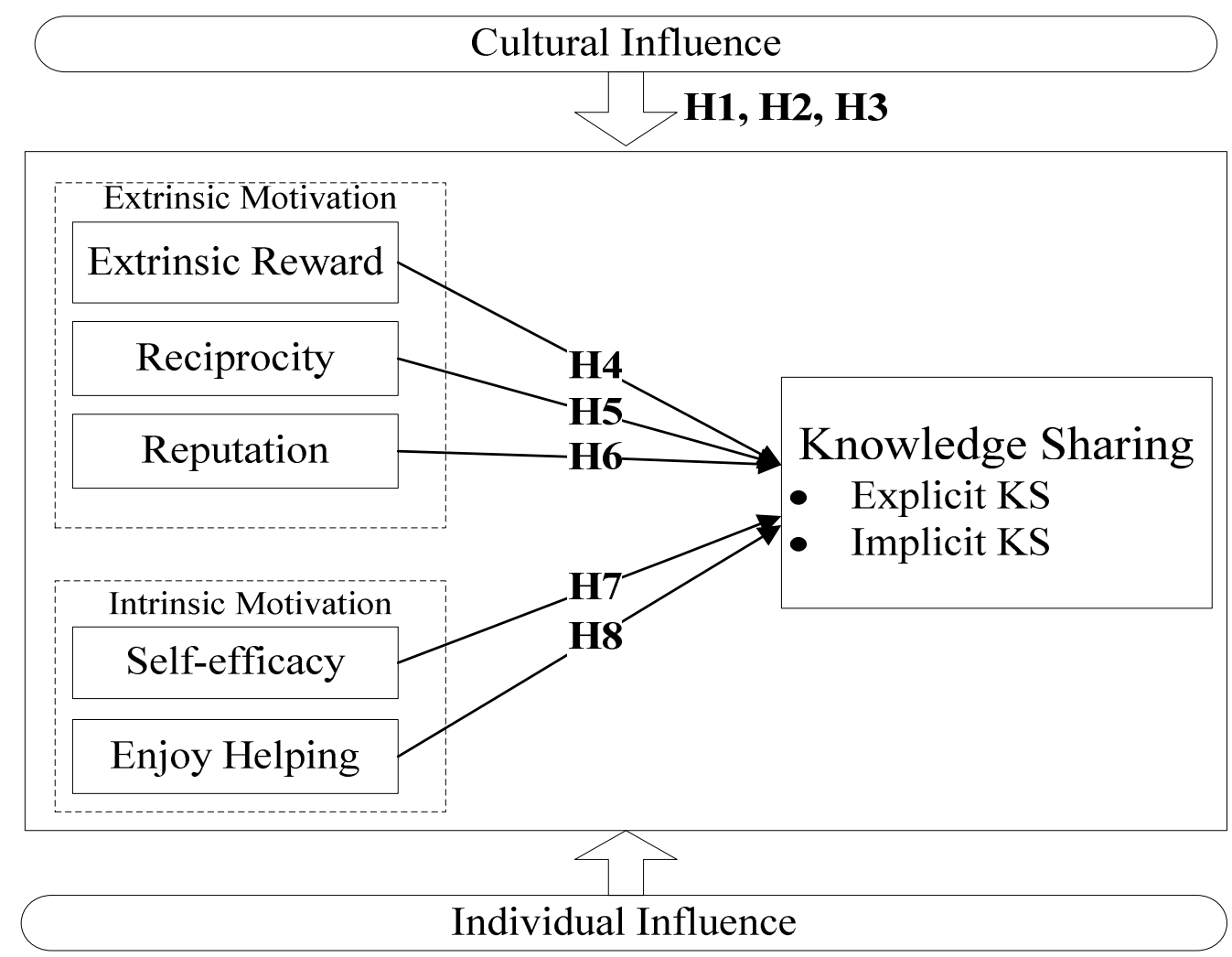

Figure 1. Research Model

\section{RESEARCH METHOdOLOGY}

\subsection{Background}

The data collection was conducted in a virtual project program among universities, conducted by City University of Hong Kong, called HKNet. The goal of the HKNet project is to offer 
International Journal of Computer Science \& Information Technology (IJCSIT) Vol 3, No 4, August 2011

students the opportunity to work together in a virtual team for problem-based collaborative learning. HKNet involves several universities from different countries, i.e., City University of Hong Kong, Beijing University of Technology (Beijing), Eindhoven University of Technology (Netherlands) and Tilburg University (Netherlands).

Several part-time postgraduate students in four universities with different backgrounds (e.g., business and engineering) applied to join the teams of HKNet to work together on different research questions. Each HKNet team consisted of 10 to 12 students in 3 locations. There were 13 teams with each team assigned a software-related topic (e.g., "policy programs for eservice", "identity theft" or "trust models and trustworthy systems/services for the Internet") to be addressed from different geographical perspectives. In other words, all teams needed to discuss their topics from a European and Asian perspective, and then write their report in an integrated framework, rather than simply connecting different parts within the timeframe of 8 weeks.

Several technologies were encouraged to be used in the course to support the knowledge sharing process. Members could upload and download documents and post progress reports through off-the-shelf learning management system (electronic blackboard) developed by City University of Hong Kong that supports forums for each team. HKNet also encourages members to communicate with each other in direct ways, such as using e-mail, google.doc, instant messengers (e.g., MSN) and VoIP technology (e.g., Skype). Videoconferencing system was thus used at the start of the project, the fourth week to report the progress, and at the end of the project.

\subsection{Measurement}

A cross-sectional survey instrument was designed to get information about all of the variables. We adapted existing scales to enhance validity. Seven items for "knowledge sharing" (KS) were adapted from the work of Lee [23] (e.g. "I share work reports and documents with members of my virtual team"), including explicit and implicit knowledge sharing measurements. Five items for individual differences ("exchange ideology", ID) were adapted from the work of Eisenberger [10] (e.g. "How hard a team member works should be affected by how well the team treats him or her" and "The failure of the team to appreciate a member's contribution should affect how hard he or she works"). Items for economic strategies ("extrinsic reward", ES, e.g. "I can get higher grades when I share my knowledge"), social reciprocal benefits ("reciprocity", SR, e.g. "When I share my knowledge, I believe that my queries for knowledge will be answered in the future"), social reputation ("reputation", SI, e.g. "Sharing my knowledge improves other team members' recognition of me"), enjoyment in helping other and self-efficacy were adapted from the work of Kankanhalli et al. [7]. Some questions were modified to match the background of this study. All the constructs were measured through seven-point scales anchored from "strongly disagree" to "strongly agree" or "never" to "very frequently".

\subsection{Data Collection}

The period of data collection was over 3 months. As participants were distributed in 3 places, an online survey was conducted. We transferred the original questionnaire to an online version using the "survey design" function of electronic blackboard. The participants of HKNet were asked to access electronic blackboard with their student or staff ID, to fill out the questionnaires 
International Journal of Computer Science \& Information Technology (IJCSIT) Vol 3, No 4, August 2011

and give comments. The online survey process was divided into three steps: 1) participants were asked whether they would like to fill out the online questionnaires, 2) participants who had agreed to fill out the questionnaire were sent the linkage of the online questionnaire, and were invited to fill them out via the website by a deadline, and 3) the status of completing questionnaires was checked with each ID, and students who had not completed the questionnaires were asked to do so. In the end, all data were saved in an Excel file.

From the 13 virtual project teams that participated in the survey, 147 participants were invited to fill out the questionnaires via the website; 113 completed responses were obtained, yielding a response rate of $76.9 \%$. Incomplete questionnaires were excluded from the data. All participants were part-time Master's students in Business and Engineering. The participants were comprised of $80.5 \%$ males and $19.5 \%$ females. Out of the 113 respondents, 10 were between 18-21 years of age (8.8\%), 80 in the 22-25 age group (70.8\%), 12 in the 26-29 age group (10.6\%), 5 in the 30-33 age group (4.4\%), 3 in the 34-37 age group (2.7\%), 1 in the 3841 group $(0.9 \%)$, and 2 were older than $41(1.8 \%)$. For work experience, the distribution is: $<1$ year $34.5 \%$, 1-4 years $46.0 \%, 5-8$ years $10.6 \%$, 9-12 years $4.4 \%$, and $>=13$ years $4.4 \%$. Regarding nationalities, 26 respondents came from Mainland China (23.0\%), 53 from Hong Kong (46.9\%), 34 from The Netherlands and other nearby countries (31.1\%).

\section{Data Analysis And Result}

\subsection{Measurement Analysis}

We assessed the reliability of all independent variables by calculating Cronbach's alpha at the individual level. As shown in Table 2, all Cronbach's alpha values were greater than 0.7, the threshold suggested by Nunnally [38].

The items were tested for validity using factor analysis with principle components analysis and varimax rotation. Factor analysis yielded 5 components with eigenvalues above 1 . These 8 components corresponded to the 8 constructs. As Tabachnick and Fidell's [39] suggestion, most of loadings are higher than acceptable level (0.32). The discriminant and convergent validity were ensured.

Table 2. Reliability of Constructs

\begin{tabular}{|l|c|c|}
\hline Construct & Number of Questions & Cronbach's Alpha \\
\hline Knowledge Sharing & 7 & 0.85 \\
\hline$\bullet \quad$ Explicit Knowledge Sharing & 4 & 0.72 \\
\hline$\bullet \quad$ Implicit Knowledge Sharing & 3 & 0.85 \\
\hline Exchange Ideology & 5 & 0.73 \\
\hline Extrinsic Reward & 4 & 0.80 \\
\hline Reputation & 5 & 0.92 \\
\hline Reciprocity & 4 & 0.78 \\
\hline Self-efficacy & 3 & 0.71 \\
\hline Enjoyment in Helping Other & 4 & 0.88 \\
\hline
\end{tabular}


International Journal of Computer Science \& Information Technology (IJCSIT) Vol 3, No 4, August 2011

\subsection{Hypotheses Tests}

\subsubsection{Cultural Influence}

We have divided sample into three subgroups, i.e., Hong Kong (sample 26), Beijing (sample 53) and Netherlands (sample 34) group. In this study, multiple regressions were used for testing hypotheses. The results are illustrated in Figure 2, 3, 4.

Based on the Figure 2, in Hong Kong group, extrinsic motivations (extrinsic reward, reciprocity and reputation) have significant influence on explicit knowledge sharing and implicit knowledge sharing. Thus, Hypothesis 1 is supported.

Based on the Figure 3, in Beijing group, intrinsic motivations (self-efficacy and enjoy helping) have significant influence on explicit knowledge sharing and implicit knowledge sharing. Thus, Hypothesis 2 is supported.

Based on the Figure 4, in Netherland group, motivations may have not any significant influence except enjoymeng in helping others on implicit knowledge sharing. Thus, Hypothesis 3 is supported.
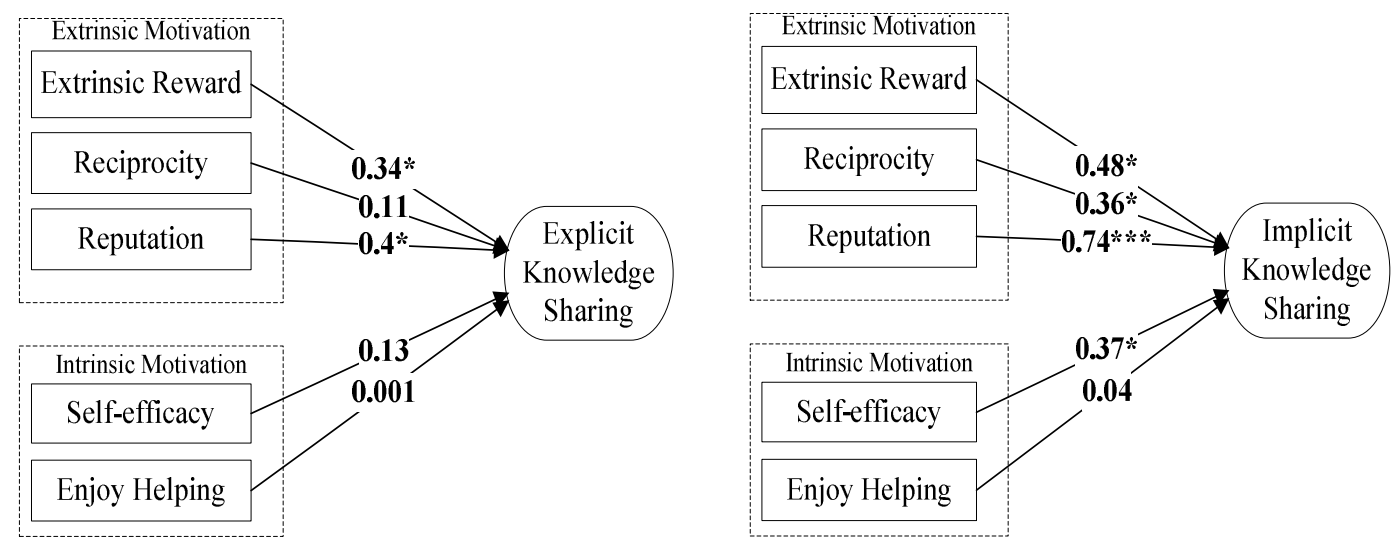

Figure 2. Hong Kong Group (Sample 26)
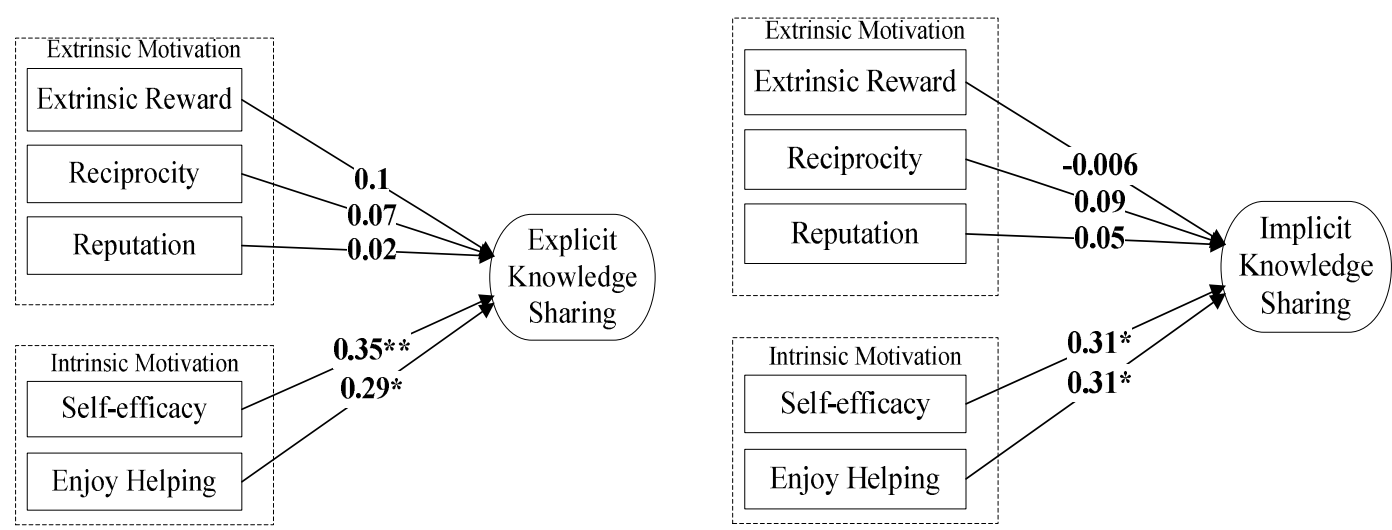

Figure 3. Beijing Group (Sample 53) 
International Journal of Computer Science \& Information Technology (IJCSIT) Vol 3, No 4, August 2011
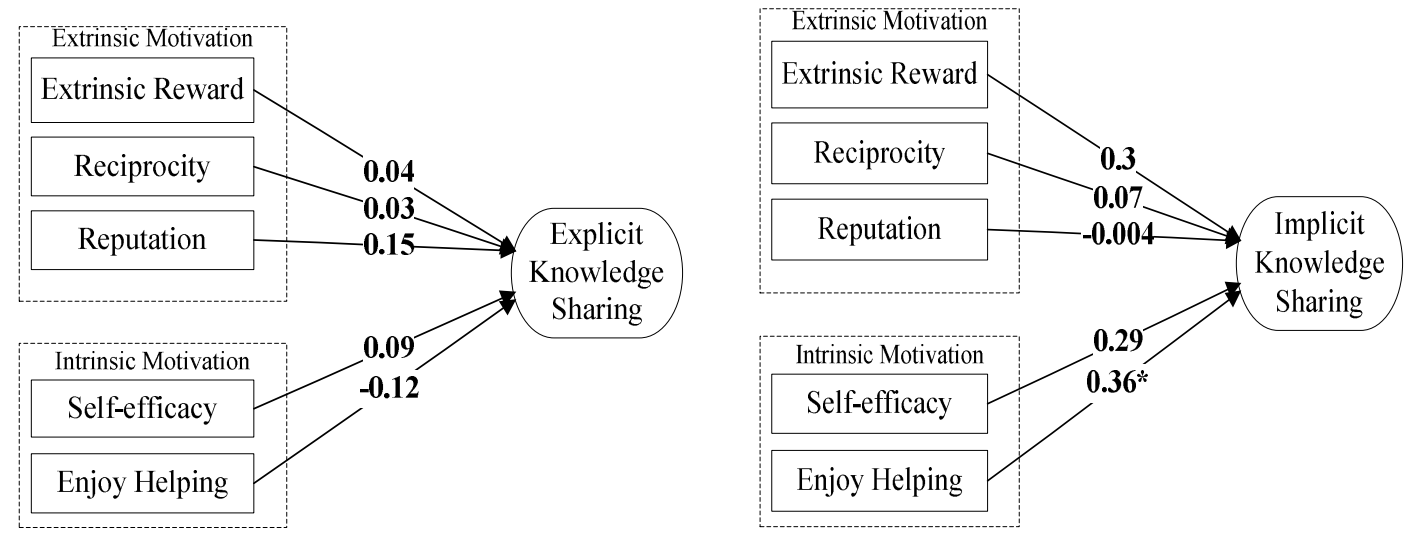

Figure 4. Netherlands Group (Sample 34)

\subsubsection{Individual Influence}

In this study, moderated multiple regression was used for testing interaction effects. Interaction terms are computed by multiplying two independent constructs. A significant change in explanatory power between the two steps indicates the presence of moderating effects. The procedure of hypotheses tests is: 1) the control variables (gender and age) were entered in the first step of regression; 2) the independent constructs were added in the second step; 3) the interaction terms were entered in the third step of regression. The Results are illustrated in Figure 5.
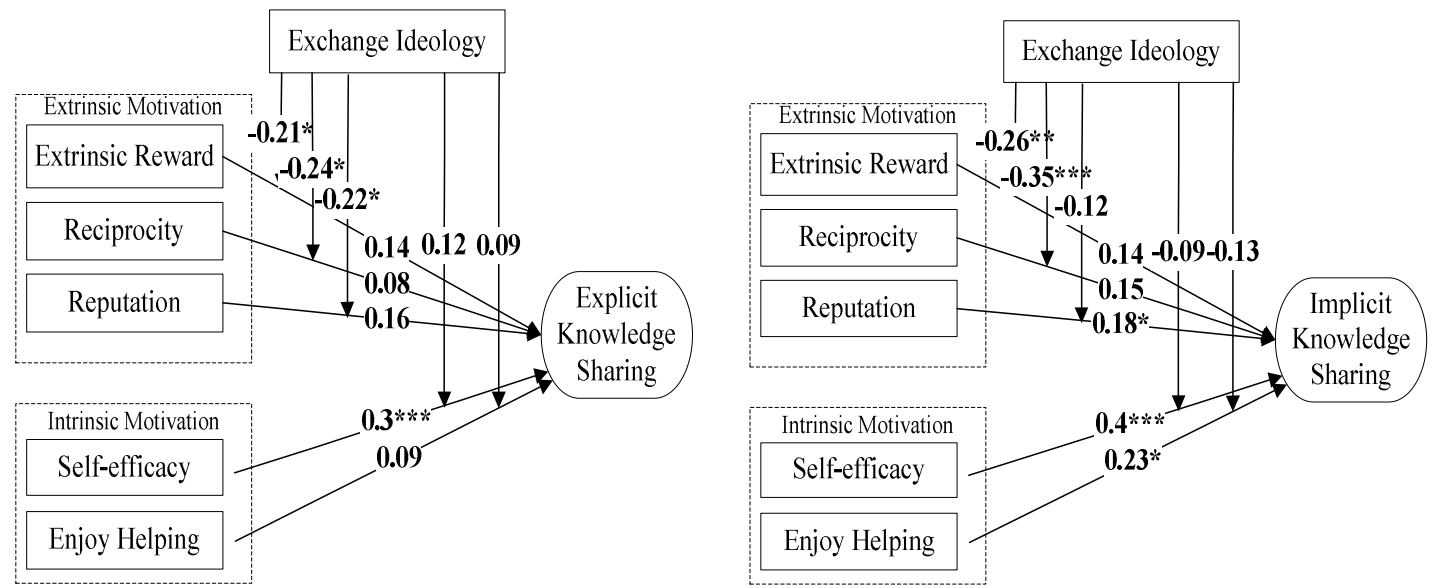

Figure 5. Results of Moderated Multiple Regression

Based on the results, the exchange ideology-extrinsic reward (ID $\times$ ES) interaction was significantly negative in the extrinsic motivation-knowledge sharing regression (i.e., explicit $\mathrm{KS}$ and implicit KS), and $\mathrm{H} 4$ was supported. The exchange ideology-reciprocity $(\mathrm{ID} \times \mathrm{SR})$ interaction was significantly negative in the extrinsic motivation-knowledge sharing regression, and $\mathrm{H} 5$ was supported. The exchange ideology-reputation $(\mathrm{ID} \times \mathrm{SI})$ was not significant, and H6 was not supported. The exchange ideology-self-efficacy was not significant in the intrinsic 
International Journal of Computer Science \& Information Technology (IJCSIT) Vol 3, No 4, August 2011

motivation-knowledge sharing regression, and $\mathrm{H} 7$ was supported. Finally, the exchange ideology-enjoy helping was not significant in the intrinsic motivation-knowledge sharing regression, and $\mathrm{H} 8$ was supported.

\section{DISCUSSION}

Based on the results of our study (Figure 2, 3,4), the findings suggest that national or regional culture may impact participants' attitude on sharing knowledge. For Hong Kong participants under culture with low uncertainty avoidance, they seem more responsive to extrinsic benefits on knowledge sharing. For Beijing participants under culture with high power distance, they seem more responsive to intrinsic benefits, while Netherlands participant may not be significantly motivated by most extrinsic and intrinsic benefits, except enjoyment by themselves.

Based on these results, the finding does not support hypothesis 6 . This may be due to the fact that members might not have enough time to gather sufficient information about their coworkers in order to establish mutual trust [40]. There is an interaction effect of reputation and mutual trust in the social exchange relationship [41]. In the teams without mutual trust, a high perception of reputation might not enhance individual KS behavior. In HKNet, each project lasted about 8 to 10 weeks. Before the project, members of the virtual teams did not know each other; after the project, they might not meet each other again. Under such circumstances, if members share critical knowledge, they may be apprehensive. However, it requires a long time to establish mutual trust. With a timeframe of 8 to 10 weeks, the interaction time may have been too short to establish considerable mutual trust among members. Moreover, the technology-based communication in virtual teams replaces face-to-face communication in organizations which can lead to a lack of mutual trust among team members and reduce the positive influence of reputation on peoples' willingness to share knowledge [42, 43]. This reputation-KS relationship is not moderated by the individual exchange ideology, and thus H6 is not supported.

This study also shows that individual exchange ideology (ID) negatively moderates the relationship between perceived benefits and knowledge sharing (KS) in the virtual teams. That is, members with strong ID show little or no KS behavior in response to economic or social benefits. A reasonable explanation for this negative moderating effect can be found in research on the exchange nature of social exchange [23, 44]. Redman et al. [23] suggest that to the extent to which employees repay their organization in an organization-employee exchange relationship, the moderating effects of exchange ideology may depend on different organizational contexts. Therefore, it is expected that the interaction effect of exchange ideology and reward may be conditional upon a third-level contextual variable. Based on these findings, there are some following extended research questions in the future studies: How can we create environment or design strategies to support knowledge sharing in the multi-cultural virtual teams?

\section{IMPLICATIONS}

This study contributes to the KS literature. It provides a more complete understanding of knowledge sharing relationship in virtual teams from the perspective of national culture and 
International Journal of Computer Science \& Information Technology (IJCSIT) Vol 3, No 4, August 2011

individual influences, demonstrating that several economic and social supporting strategies have different influences in different cultural orientation, and negatively interact with individual differences on participants' KS behaviors. Some implications are provided as the following. First, participants' cultural orientation should be considered in establishing the virtual teams, especially for multi-national virtual teams. An incentive scheme should be introduced to encourage individuals to share knowledge with the rules about "different strokes for different folks"- a portfolio of incentives should be provided to different individuals for everyone favoring the outcomes of their sharing effort. Second, exchange ideology could be seen as the important indicator of individual difference in selecting members of virtual teams. In contrary to long-term project, virtual teams dealing with short-term projects may choose the individuals with low exchange ideology. Second, some strategies should be considered to reducing the negative influence of exchange ideology. It is supposed that the moderating effect of reciprocity is negative due to the short-term project. Management of virtual teams should encourages members to establish longer social relationship through informal ways, such as communicating and adding friend through software such as instant messenger and VoIP technology.

\section{LIMITATIONS AND FUTURE STUDY}

This section discusses the potential limitations from the perspective of threats to validity of this study. The threats to validity include: internal validity, and external validity.

\subsection{Internal Validity}

Internal validity refers to whether there is causal relationship between the independent variables (IVs) and dependent variables (DVs) measured [45]. In this study, there are two possible threats to internal validity. First, the data analysis techniques (e.g., moderated multiple regression) applied in this study cannot do "feedback" analysis to ensure the casual relationship between IVs and DVs. Second, the data analysis based on the cross-sectional data cannot provide the real causal relationship between IVs and DVs. The use of cross-sectional survey is also a potential limitation in this study.

However, we applied several strategies to reduce the threat to internal validity. For example, we designed the research models by adopting well-established theories which provide clear causal relationships between constructs. Furthermore, considering the new research model established in this study, the cross-sectional survey can be applied as an exploratory tool to test the relationship for the new theory. Thus, the threats of internal validity in this study are not very serious. One possible way to ensure internal validity is in applying longitudinal studies to test directions of causality. Future studies could apply longitude studies to re-test research models to investigate the directions of causality.

\subsection{External Validity}

External validity refers to whether the findings in one research can be generalized to different populations of persons, times and organizations [45]. In this study, there are several possible threats to external validity. First, we collected data from part-time post-graduates in universities. However, we would argue using part-time post-graduate students with work experience, rather than those without work experience, helps facilitate improved external validity [46]. 
International Journal of Computer Science \& Information Technology (IJCSIT) Vol 3, No 4, August 2011

\section{ACKNOWLEDGMENT}

The work described in this paper was supported by the Project Funds for Youth, Institute of Policy and Management, Chinese Academy of Sciences (No: O900971J01).

\section{REFERENCES}

[1] M. Alavi and D. E. Leidner, "Knowledge Management and Knowledge Management Systems: Conceptual Foundations and Research Issues," MIS Quarterly, vol. 25, pp. 107-136, 2001.

[2] I. K. Hejduk, "On the Way to the Future: The Knowledge-Based Enterprise," Human Factors and Ergonomics in Manufacturing, vol. 15, pp. 5-14, 2005.

[3] K. Breu and C. J. Hemingway, "Making organizations virtual: the hidden cost of distributed teams," Journal of Information Technology, vol. 19, pp. 191-202, 2004.

[4] V. Ratcheva, "Enabling processes for sharing knowledge in virtual business relationships," International Journal of Internet and Enterprise Management, vol. 1, pp. 360-372, 2003.

[5] M. Alavi and D. E. Leidner, "Knowledge Management Systems: Issues, Challenges and Benefits," Communications of AIS, vol. 2, pp. 1-37, 1999.

[6] D. Snowden, "The Knowledge You Need, Right When you Need it," Knowledge Management Review, vol. 5, pp. 24-27, 2003.

[7] A. Takkinen, "Knowledge Sharing in Virtual Teams: Action Research," in Computer Science and Information Systems. vol. Master Jyvaskyla: University of Jyvaskyla, 2004, pp. 11-16.

[8] G. Hertel, S. Geister, and U. Konradt, "Managing virtual teams: A review of current empirical research," Human Resource Management Review, vol. 15, pp. 69-95, 2005.

[9] A. Kankanhalli, B. Tan, and K. K. Wei, "Contributing Knowledge to Electronic Knowledge Repositories: An Emprical Investigation," MIS Quarterly, vol. 29, pp. 113-143, 2005.

[10]H. F. Lin, "Effects of extrinsic and intrinsic motivation on employee knowledge sharing intentions," Journal of Information Science, vol. 33, pp. 135-149, 2007.

[11] G. W. Bock and Y. G. Kim, "Breaking the Myths of Rewards: An Exploratory Study of Attitudes about Knowledge Sharing," Information Resources Management Journal, vol. 15, pp. 14-21, 2002.

[12] G. W. Bock, R. W. Zmud, Y. G. Kim, and J. N. Lee, "Behavioral Intention Formation in Knowledge Sharing: Examining the Roles of Extrinsic Motivators, Social-Psychological Forces, and Organizational Climate," MIS Quarterly, vol. 29, pp. 87-111, 2005.

[13]R. Cropanzano and M. S. Mitchell, "Social Exchange Theory: A Interdisciplinary Review," Journal of Management, vol. 31, pp. 874-900, 2005.

[14]T. H. Davenport and L. Prusak, Working Knowledge: How Organizations Manage What They Know. Boston: Harvard Business School Press, 1998.

[15]K. M. Bartol and A. Srivastava, "Encouraging Knowledge Sharing: The Role of Organizational Reward Systems," Journal of Leadership and Organization Studies, vol. 9, pp. 64-76, 2002.

[16] A. Kankanhalli, B. C. Y. Tan, K. K. Wei, and M. C. Holmes, "Cross-cultural Differences and Information Systems Developer Values," Decision Support Systems, vol. 38, pp. 183-195, 2004. 
International Journal of Computer Science \& Information Technology (IJCSIT) Vol 3, No 4, August 2011

[17] B. C. Y. Tan, K. K. Wei, D. L. Watson, and E. R. Clapper, "Computer-mediated Communication and Majority Influence:Assessing the Impact in an Individualistic and a Collectivistic Culture," Management Science, vol. 44, 1998.

[18] D. P. Ford, C. E. Connelly, and D. B. Meister, "Information Systems Research and Hofstede's Culture's Consequences: An Uneasy and Incomplete Partnership," IEEE Transaction on Engineering Managment, vol. 50, pp. 8-25, 2003.

[19] G. Hofstede, Cultures and Organizations:Software of the Mind. London: McGraw-Hill, 1991.

[20] A. H. Smith, Chinese Characteristics: Simon Publications, 2001.

[21]R. Eisenberger, R. Huntington, S. Hutchison, and D. Sowa, "Perceived Organizational Support," Journal of Applied Psychology, vol. 71, pp. 500-507, 1986.

[22]L. A. Witt, "Exchange Ideology as a Moderator of Job Attitudes-Organizational Citizenship Behaviors Relationships," Journal of Applied Social Psychology, vol. 21, pp. 1490-1501, 1991.

[23] T. Redman and E. Snape, "Exchange Ideology and Member-Union Relationships: An Evaluation of Moderation Effects," Journal of Applied Psychology, vol. 90, pp. 765-773, 2005.

[24]I. Nonaka and H. Takeuchi, The knowledge Creating Company: How Japanese Companies Create the Dynamics of Innovation. New York: Oxford Uiversity Press, 1995.

[25]F. Nickols, "The Knowledge in Knowledge Management," in The Knowledge Management Yearbook 2000-2001, J. W. Cortada and J. A. Woods, Eds.: Butterworth-Heinemann Ltd, 2001.

[26] J. N. Lee, "The impact of knowledge sharing, organizational capability and partnership quality on IS outsourcing success," Information \& Management, vol. 38, pp. 323-335, 2001.

[27]C. F. Fey and P. Furu, "Top Management Incentive Compensation and Knowledge Sharing in Multinational Corporations," Strategic Management Journal, vol. 29, pp. 1301-1323, 2008.

[28]D. J. Lee and J. H. Ahn, "Reward Systems for Intra-Organizational Knowledge Sharing," European Journal of Operational Research, vol. 180, pp. 938-956, 2007.

[29] C. Wolfe and T. Loraas, "Knowledge Sharing: The Effects of Incentives, Environment, and Person," Journal of Information Systems, vol. 22, pp. 53-76, 2008.

[30] P. M. Blau, Exchange and Power in Social Life. New York: Wiley, 1964.

[31] L. D. Molm, Coercive Power in Social Exchange. New York: Cambridge University Press, 1997.

[32] A. Cabrera and E. F. Cabrera, "Knowledge Sharing Dilemmas," Organization Studies, vol. 23, pp. 687-710, 2002.

[33]E. Carmel, Global Software Teams:Collaborating Across Borders And Time Zones: Prentice Hall PTR, 1999.

[34] J. Brockner, Y.-R. Chen, E. A. Mannix, K. Leung, and D. P. Skarlicki, "Culture and Procedural Fairness: When the Effects of What You Do Depend on How You Do it," Administrative Science Quarterly, vol. 45, pp. 138-159, 2000.

[35] V. U. Druskat and D. C. Kayes, "Learning versus Performance in Short-Term Project Teams," Small Group Research, vol. 31, pp. 328-353, 2000.

[36] A. Rutkowski, C. Saunders, D. Vogel, and M. Genuchten, "Is it Already 4 AM in your Time Zone? Focus Immersion and Temperal Dissociation in Virtual Teams," Small Group Research, vol. 38, pp. 98-129, 2007.

[37]C. Saunders, C. Van Slyke, and D. Vogel, "My Time or Yours? Managing Time Vision in Global Virtual Teams," Academy of Management Executive, vol. 18, pp. 19-31, 2004.

[38] J. C. Nunally, Psychometric Theory. New York: McGraw-Hill, 1978. 
International Journal of Computer Science \& Information Technology (IJCSIT) Vol 3, No 4, August 2011

[39] B. G. Tabachnick and L. S. Fidell, Using Multivariate Statistics (4th ed.). Upper Saddle River, NJ: Allyn \& Bacon, 2000.

[40]P. Kanawattanachai and Y. Yoo, "Dynamic nature of trust in virtual teams," Journal of Strategic Information Systems, vol. 11, pp. 187-213, 2002.

[41]R. Bennett and H. Gabriel, "Reputation, Trust and Supplier Commitment: The Case of Shipping Company/Seaport Relations," Journal of Business \& Industrial Marketing, vol. 16, pp. 425-438, 2001.

[42] L. L. Martins, L. L. Gilson, and M. T. Maynard, "Virtual Teams: What Do We Know and Where Do We Go From Here?," Journal of Management, vol. 30, pp. 805-835, 2004.

[43] A. Powell, G. Piccoli, and B. Ives, "Virtual Teams: A Review of Current Literature and Directions for Future Research," The DATABASE for Advances in Information Systems, vol. 35, 2004.

[44] R. R. Sinclair and L. E. Tetrick, "Social Exchange and Union Commitment: A Comparison of Union Instrumentality and Union Support Perceptions," Journal of Organizational Behavior, vol. 16, pp. 669-680, 1995.

[45] M. Cook and D. T. Campbell, Quasi-Experimentation: Design and Analysis Issues for Field Settings. Boston: Houghton Mifflin, 1979.

[46]T. A. Scandura and E. A. Williams, "Research Methodology in Management: Current Practices, Trends, and Implications for Future Research," Academy of Management Journal, vol. 43, pp. $1248-1264,2000$. 